\title{
BMJ Open Haemodynamic effects of a prehospital emergency anaesthesia protocol consisting of fentanyl, ketamine and rocuronium in patients with trauma: a retrospective analysis of data from a Helicopter Emergency Medical Service
}

\author{
Ewoud ter Avest (D) , ${ }^{1,2}$ Dassen Ragavan, ${ }^{1}$ Joanne Griggs, ${ }^{1,3}$ Michael Dias, ${ }^{1}$ \\ Sophie A Mitchinson, ${ }^{1}$ Richard Lyon ${ }^{1,4}$
}

To cite: ter Avest E, Ragavan D, Griggs J, et al. Haemodynamic effects of a prehospital emergency anaesthesia protocol consisting of fentanyl ketamine and rocuronium in patients with trauma: a retrospective analysis of data from a Helicopter Emergency Medical Service. BMJ Open 2021;11:e056487. doi:10.1136/ bmjopen-2021-056487

- Prepublication history and additional supplemental material for this paper are available online. To view these files, please visit the journal online (http://dx.doi.org/10.1136/ bmjopen-2021-056487).

EtA and DR contributed equally.

Received 20 August 2021 Accepted 26 November 2021

Check for updates

(C) Author(s) (or their employer(s)) 2021. Re-use permitted under CC BY-NC. No commercial re-use. See rights and permissions. Published by BMJ.

For numbered affiliations see end of article.

Correspondence to

Dr Ewoud ter Avest;

e.ter.avest@umcg.nl

\section{ABSTRACT}

Objectives Prehospital rapid sequence induction (RSI) of anaesthesia is an intervention with significant associated risk. In this study, we aimed to investigate the haemodynamic response over time of a prehospital RSI protocol of fentanyl, ketamine and rocuronium in a heterogeneous population of trauma patients.

Design, setting and participant We performed a retrospective study of all trauma patients who received a prehospital RSI for trauma by a physician staffed Helicopter Emergency Medical Service in the UK between 1 June 2018 and 1 February 2020.

Primary outcome measure Primary outcome was defined as the incidence of clinically relevant hypotensive (systolic blood pressure (SBP) or mean arterial pressure (MAP) $>20 \%$ below baseline, with an absolute SBP $<90 \mathrm{~mm} \mathrm{Hg}$ or MAP $<65 \mathrm{~mm} \mathrm{Hg}$ ) or hypertensive (SBP or MAP $>20 \%$ above baseline) episodes in the first 10 minutes post-RSI.

Results In total, 322 patients were included. 204 patients (63\%) received a full-dose induction of $3 \mu \mathrm{g} / \mathrm{kg}$ fentanyl, $2 \mathrm{mg} / \mathrm{kg}$ ketamine and $1 \mathrm{mg} / \mathrm{kg}$ rocuronium, whereas 128 patients (37\%) received a reduced-dose induction. Blood pressures decreased on average $12 \mathrm{~mm} \mathrm{Hg}(95 \% \mathrm{Cl} 7$ to 16$)$ in the full-dose group and $6 \mathrm{~mm} \mathrm{Hg}(95 \% \mathrm{Cl} 1$ to 11 ) in the reduced-dose group, $p=0.10$ ). A hypotensive episode (mean SBP drop $53 \mathrm{~mm} \mathrm{Hg}$ ) was noted in 29 patients: $17(8.3 \%)$ receiving a full dose and $12(10.2 \%)$ receiving a reduced-dose induction, $p=0.69$. The blood pressure nadir was recorded on average 6-8 min after RSI. A hypertensive episode was present in 22 patients $(6.8 \%)$. The highest blood pressures were recorded in the first 3 min after RSI.

Conclusion Prehospital induction of anaesthesia for trauma with fentanyl, ketamine and rocuronium is not related to a significant change in haemodynamics in most patients. However, a (delayed) hypotensive response with a significant drop in SBP should be anticipated in a minority of patients irrespective of the dose regimen chosen.
Strengths and limitations of this study

To the best of our knowledge, this is the first study to evaluate the haemodynamic effects of a prehospital rapid sequence induction (RSI) protocol for trauma induction of anaesthesia comprising of fentanyl, ketamine and rocuronium by accurate high frequency haemodynamic measurements.

- The study was conducted by a physician staffed Helicopter Emergency Medical Services in a large and heterogeneous cohort of trauma patients.

- Given the retrospective design of the study, only associations and no causal relationships could be reported between the medication administered during the RSI process and changes in blood pressures.

\section{INTRODUCTION}

Rapid Sequence Induction (RSI) of anaesthesia can result in significant hypoxia and hypotension during and after the procedure in critically injured patients. ${ }^{1-5}$ This is especially true when RSI is performed in the prehospital setting, where resources and equipment are often limited and intubation conditions are often suboptimal. ${ }^{6}$ Major trauma patients often have injuries or medical conditions resulting in deranged physiology and/or anatomy which can complicate the procedure.

Many Helicopter Emergency Medical Services (HEMS) have developed standard operating procedures (SOPs) and checklists for the conduct of pre-hospital RSI. ${ }^{7-9}$ The purpose of these is to help deliver RSI in a uniform, safe manner, to reduce the potential for human error and to mitigate the risks of prehospital RSI as much as possible. Prehospital RSI is usually performed 
using an anaesthetic induction agent, a neuromuscular blocker and often an opioid to attenuate the sympathomimetic response to laryngoscopy. The combination of rocuronium (as the neuromuscular blocker of choice), ketamine and fentanyl is frequently used and has been shown to produce favourable intubation conditions in the vast majority of trauma patients. ${ }^{6}{ }^{10}$ Limited information, however, is available about the haemodynamic safety, as sample sizes in previous studies were relatively small and high frequency sampling of haemodynamic measurements was not performed. ${ }^{10} 11$ Therefore, in this study, we aimed to investigate the haemodynamic response over time to a prehospital RSI protocol of fentanyl, ketamine and rocuronium in a heterogeneous population of trauma patients, attended by a physician-staffed HEMS service.

\section{METHODS}

\section{Study setting and design}

We performed a retrospective study of all patients who received a prehospital RSI for traumatic injuries by Air Ambulance Kent Surrey and Sussex (AAKSS) between 1 June 2018 (date of introduction of HEMSBASE V.2.0 electronic patient clinical record system) and 1 February 2020 (date of last Trauma Audit Research Network outcome data availability at the time of data abstraction on 1 May 2020). We investigated how haemodynamics were affected in the postanaesthetic period and how this was related to drug, dose and timing of administration of the RSI drugs, in the study cohort as a whole and in predefined subgroups, characterised by specific patient and injury characteristics.

AAKSS is a HEMS service covering three counties in the southeast of England, with a resident population of 4.5 million. Two doctor/paramedic teams respond in either a helicopter or response car from one base. Physicians have a minimum of 5 years postgraduate experience, including a minimum of 6 months hospital anaesthesia training. Paramedics undergo further specialist training, including theoretical modules on prehospital anaesthesia. All crew undergo an intense training period prior to independent practice, including structured medical education, simulation training and operational supervision by prehospital care consultants. During this period, training is focused to ensure that crews are competent in performing safe pre-hospital RSI.

\section{Conduct of RSI}

A Standard Operating Procedure (SOP) is in place to describe indications, preparation and conduct of RSI for patients with traumatic injuries. Indications include actual or impending airway compromise; ventilatory failure; unconsciousness; anticipated clinical course and for humanitarian reasons. Prior to induction of anaesthesia, the patient's position is optimised (ideally on an ambulance trolley with $360^{\circ}$ access to the patient). Necessary RSI equipment is prepared in a standard kit-dump, non-invasive monitoring is attached, and the patient is preoxygenated for at least $3 \mathrm{~min}$. For trauma induction, fentanyl is used as an opioid, ketamine as the induction agent and rocuronium as the muscle relaxant. Drugs are given in this order, one immediately after the other, without interruption or pause. At the time of the study, for trauma patients who were not grossly hypovolaemic, the standard dose of drugs used was: fentanyl $3 \mu \mathrm{g} / \mathrm{kg}$, ketamine $2 \mathrm{mg} / \mathrm{kg}$ and rocuronium $1 \mathrm{mg} / \mathrm{kg}$ (3-2-1), with suggested reductions in fentanyl and ketamine doses in patients with suspected hypovolaemia and frail/elderly patients (1-1-1), and omitting of fentanyl (0-1-1) or using rocuronium only $(0-0-1)$ in critically haemodynamically unstable patients. Before, during and after RSI, noninvasive systolic blood pressure (SBP), diastolic blood pressure (DBP), mean arterial pressure (MAP) are measured at 3 min intervals and other vital signs including heart rate (HR), oxygen saturation $\left(\mathrm{SpO}_{2}\right)$ and end-tidal $\mathrm{CO}_{2}$ $\left(\mathrm{ETCO}_{2}\right)$ are captured at $1 \mathrm{~min}$ intervals by the Tempus Pro monitor, and uploaded to the dedicated electronic patient clinical record system AAKSS uses (HEMSbase V.2.0, Medic One Systems, UK), allowing numeric and visual trend analysis.

\section{Study population}

Patients were eligible for inclusion in the study if they were aged $>18$ and underwent prehospital RSI by AAKSS for traumatic injuries during the study period. Patients were excluded if they were intubated for medical reasons, patients in traumatic cardiac arrest (who were predominantly intubated without the use of medication) and patients for whom RSI data were not registered in HEMSbase for logistical or technical reasons.

\section{Clinical endpoints}

Primary endpoint was defined as the occurrence of a clinically relevant hypotensive or hypertensive response in the first $10 \mathrm{~min}$ post-RSI. A hypotensive response was defined as the occurrence of at least two measurements (to correct for spurious or artefactual readings) of SBP $>20 \%$ lower than baseline with an absolute SBP $<90 \mathrm{~mm} \mathrm{Hg}$, OR a MAP $>20 \%$ lower than baseline with an absolute MAP $<65 \mathrm{~mm} \mathrm{Hg}$. A hypertensive response was defined as two or more measurements of SBP or MAP $>20 \%$ above baseline.

Secondary endpoints were the difference in pre-RSI and post-RSI blood pressures in the first $10 \mathrm{~min}$ post-RS, and the timing of occurrence of hypotensive and hypertensive episodes in relation to RSI.

\section{Data acquisition}

The following data were retrieved from the HEMSbase electronic patient record: patient descriptors (age, gender, estimated weight), mission timings (emergency call time, HEMS arrival on scene time, RSI time, arrival at hospital time); injury descriptors (mechanism of injury, injured body regions, suspected critical 'Code Red' major haemorrhage); clinical findings on arrival of HEMS (GCS, 
presence of a central or radial pulse); indication for RSI; pre-RSI treatments (pre-RSI fluids, blood products, medication, preoxygenation); conduct of RSI (laryngoscopy view grade, first pass success rate, overall success rate, rescue techniques used); RSI-drug administration (type, dosing regimen, route of administration); and all pre-RSI and post-RSI vital signs ( $\mathrm{SBP}, \mathrm{DBP}, \mathrm{HR}, \mathrm{ETCO}_{2}$ and $\mathrm{SpO}_{2}$ ) in the $10 \mathrm{~min}$ before and after RSI.

\section{Calculations}

Baseline (pre-RSI) SBP, MAP, HR, $\mathrm{SpO}_{2}$ and $\mathrm{ETCO}_{2}$ were calculated as the average of all measurements conducted for these variables within the last $10 \mathrm{~min}$ prior to RSI. Pre-RSI shock index was calculated as the mean pre-RSI HR/ mean pre-RSI SBP. Mean post-RSI HR and blood pressures were calculated as the average of all measured blood pressures within the first $10 \mathrm{~min}$ post-RSI. The absolute change in blood pressure after RSI was calculated by subtracting the mean baseline blood pressures from the mean post-RSI blood pressures. To analyse trends over time in blood pressures (and thereby the timing of the maximum blood pressure decrease or increase and the timing of hypotensive and hypertensive episodes), a data imputation algorithm based on next nearest values was used to account for missing data points, as blood pressure was measured every $3 \mathrm{~min}$ in all patients, but not for all patients at the same time within the $10 \mathrm{~min}$ interval post-RSI.

\section{Patient and public involvement}

Lay representation on the AAKSS charity board expressed support for further research being undertaken into prehospital RSI. Patients were not directly involved in the design, recruitment or conduct of the study. Results and impact of the study will be shared with the lay representatives.

\section{Statistical analysis}

Categorical data are reported as frequency (n) and percent $(\%)$ and numerical data as mean (SD) or median (IQR) (depending on whether data were normally distributed or not). One sample Kolmogorov-Smirnov test was used to test for normality. Patients were categorised into two dose regimes based on the medication they received for their RSI: a full-dose regime (3-2-1) and a reduceddose regime (1-1-1, 0-1-1,0-0-1). For categorical data, including the primary endpoint (number of hypotensive and hypertensive episodes) was compared between the two dose regimes using Fisher's exact test. Mann-Whitney $\mathrm{U}$ test or Student's t-tests were used as appropriate to compare numerical data. Absolute changes in HR, SBP and MAP over time were analysed using a paired t-test. A linear mixed model analysis was performed to look for time-to-group interactions for the effect of induction (full-dose or reduced dose) on SBP and MAP in the first 10 min after RSI. Missing data were reported in the results section according to the Strengthening the Reporting of Observational Studies in Epidemiology guideline. ${ }^{12}$
Statistical significance was set as a two-tailed $\mathrm{p}$ value $<0.05$. All statistical analyses were conducted using SPSS V.26.0 for Apple statistical package.

\section{RESULTS}

\section{Study population}

A total of 822 records were retrieved from HEMSbase V.2.0. After exclusion of accidental duplicate entries and intubations without administration of drugs, 542 RSI's remained for the study period. Of these, 151 were for a medical (ie, non-traumatic) condition. Four patients were excluded as other anaesthetic induction regimens were used. In 5 patients, the RSI time was not noted and in 60 patients the exact RSI time and timing of measured blood pressures could not be confirmed, for example, due to the use of ground ambulance service monitoring in the pre-RSI phase not being synchronised with the HEMS monitoring equipment. Further results refer to the remaining 322 patients (figure 1).

The majority of patients $(n=204,63 \%)$ received a fulldose RSI regimen (either as an exact 3:2:1 ratio $(n=183)$ or with slight adjustments to account for pre-RSI medication $(\mathrm{n}=21)$ ), whereas the remaining 118 patients received a reduced-dose regimen (exact ratio 0:0:1 $(n=7)$; exact ratio $0: 1: 1(n=18)$; exact ratio $1: 1: 1 \quad(n=71)$; or with slight adjustments from the latter to account for pre-RSI medication $(\mathrm{n}=22)$ ). Patient characteristics stratified by dosing regimen (full dose vs reduced dose) are shown in table 1. Patients receiving a reduced-dose regimen more commonly had injuries in multiple body regions and as expected, had lower pre-RSI blood pressures, and more often triggered a code red response, activating the in-hospital transfusion protocol (table 1).

\section{Conduct of RSI}

Conduct of RSI is shown in table 2. The vast majority $(75 \%)$ of subjects were anaesthetised within $30 \mathrm{~min}$ of arrival of the HEMS team on scene (mean (SD): after 24 (9) $\min$ ). Preoxygenation was standard practice for all inductions and a significant number of patients received analgesia and/or sedation prior to the RSI. Blood products and hypertonic saline were administered in a small subset of patients pre-RSI. Induction medication was primarily administered via the intravenous route. Subsequent intubation conditions were generally good (>95\% Cormack-Lehane grade I/II), resulting in a first pass intubation success rate of $93.5 \%$, and an overall intubation success rate of $99.7 \%$ ( 1 failed intubation, resolved with a successful surgical airway).

\section{Haemodynamic response to RSI}

Average baseline-RSI and post-RSI values for HR, SBP and MAP are shown in table 3. On average, SBP and MAP were, respectively, $10 \mathrm{~mm} \mathrm{Hg}$ (95\% CI 6 to 13) and $7 \mathrm{~mm} \mathrm{Hg}(95 \%$ CI 4 to 9) lower in the first $10 \mathrm{~min}$ after induction. Blood pressure reductions were more marked in the full-dose group compared with the reduced-dose 


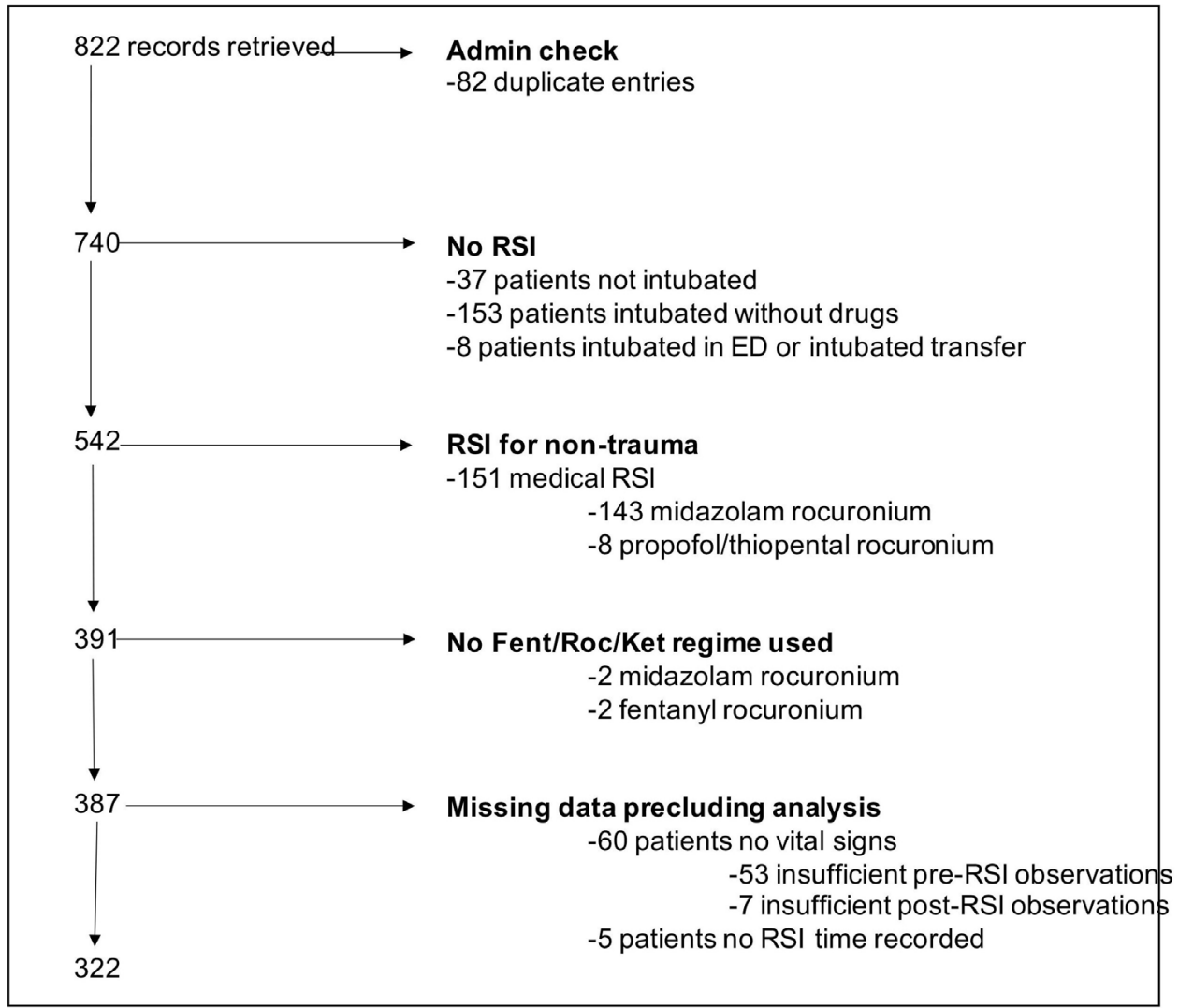

Figure 1 Derivation of study population. ED, emergency department; HEMS, Helicopter Emergency Medical Service; OHCA, Out of Hospital Cardiac Arrest; RSI, rapid sequence induction.

group: SBP 12 (95\% CI 7 to 16$) \mathrm{mm} \mathrm{Hg}$ reduction in the full-dose group versus 6 (95\% CI 1 to 11) $\mathrm{mm} \mathrm{Hg}$ in the reduced-dose group, $\mathrm{p}=0.10$, online supplemental file 1 .

A true hypotensive response was present in 29 patients, 17 of whom received a full-dose regimen (incidence $8.3 \%$ ) and 12 a reduced-dose regimen (incidence $10.2 \%$ ), $\mathrm{p}=0.69$. Of the 17 patients who received a full-dose regime, 5 had a SI>0.8 (5/43,11.6\%) and $12(12 / 161,7.5 \%)$ had a $\mathrm{SI}<0.8$ prior to RSI. A hypertensive response was present in 22 patients (16 in the full-dose group $(7.8 \%)$, and in 6 patients in the reduced-dose group $(5.9 \%)$, which was not significantly different $(\mathrm{p}=0.28)$.

Code Red patients were induced almost universally with a reduced-dose regime $(29 / 30)$. The incidence of a hypotensive response in these patients $(\mathrm{n}=3,10.0 \%)$ was similar to non-code red patients $(\mathrm{n}=26,8.9 \%, \mathrm{p}=0.74)$. Patients with isolated head injuries were induced with a full-dose regime in the majority of cases $(103 / 137)$. Eight patients $(5.8 \%)$ with isolated head injury had a hypotensive response, which was not significantly different from patients who did not have isolated head injury $(n=21$, $11.3 \%, \mathrm{p}=0.09)$. Ten patients with isolated head injury $(7.3 \%)$ had a hypertensive response (6/10 full dose, $4 / 10$ reduced dose), which was not significantly different from patients who did not have isolated head injury $(n=12$, $6.4 \%, \mathrm{p}=0.84)$.

When physicians on scene adjusted the dose from the standard induction regimes as per SOP to account for medication administered pre-RSI, this was not associated with a reduced rate of hypotensive episodes in sensitivity analysis (online supplemental file 2).

\section{Timing and severity of haemodynamic response to RSI}

The responses of SBP and MAP to RSI, plotted against time, are shown in figure 2. Minimum blood pressures were reached, on average, $8 \mathrm{~min}$ after induction in the full-dose group (figure 2A), and $6 \mathrm{~min}$ after induction in the reduced-dose group (figure 2B). Patients with a hypotensive response $(n=29)$ had an average maximum drop in SBP of $53 \mathrm{~mm} \mathrm{Hg}$, occurring $6 \mathrm{~min}$ after induction (figure 2C). Patients with a hypertensive response $(\mathrm{n}=22)$ had an average maximum increase in SBP of $29 \mathrm{~mm} \mathrm{Hg}$, occurring in the first $3 \mathrm{~min}$ after induction (figure 2D). The timing of the hypotensive and hypertensive responses was not related to the dose regimen administered: there was no time to group interaction.

\section{Long-term outcome}

One patient died before arrival in hospital due to noncompressible exsanguinating haemorrhage. Further follow-up from TARN data was available for 204 patients. $89.7 \%$ of them (93.3\% in the full-dose regimen and $84.6 \%$ in the reduced-dose regimen, $\mathrm{p}=0.056$ ) survived the first 24 hours after their injury, and $68.6 \%$ (73.9\% in the fulldose regimen and $61.3 \%$ in the reduced-dose regimen, $\mathrm{p}=0.062$ ) survived until hospital discharge. 
Table 1 Patient descriptors of patients anaesthetised with standardised dosing regimen of fentanyl, ketamine and rocuronium

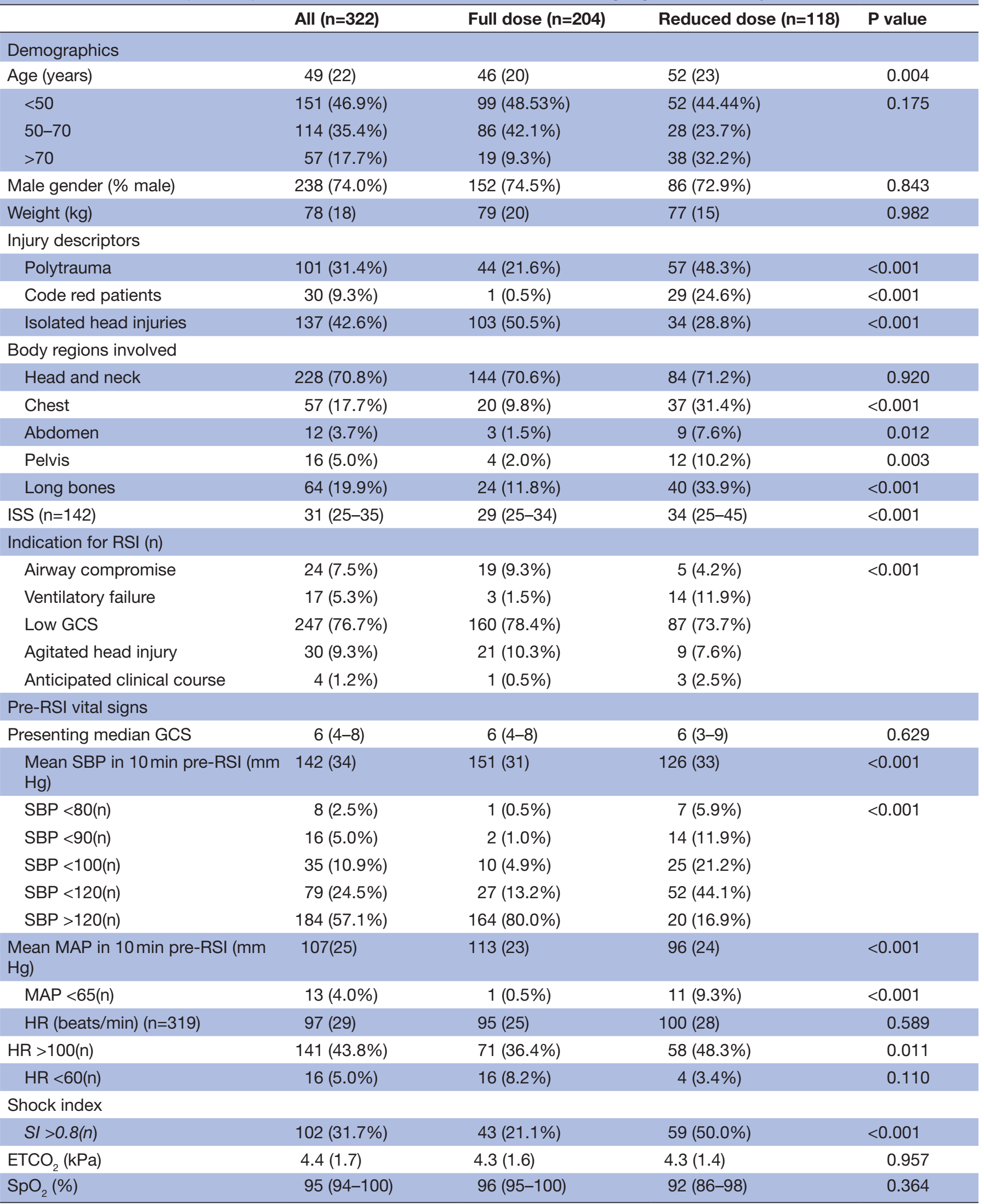

Categorical data are reported as frequency $(\mathrm{n})$ and percentage (\%) and numerical data as median (IQR) or mean (SD). ETCO2, end tidal CO2; GCS, Glasgow Coma Scale; MAP, mean arterial pressure; SBP, systolic blood pressure; SI, Shock Index; SpO2, oxygen saturation. 
Table 2 RSI descriptors and outcome of patients anaesthetised with standardised dosing regimen of fentanyl, ketamine and rocuronium

\begin{tabular}{|c|c|c|c|c|}
\hline & All $(n=322)$ & Full dose (n=204) & $\begin{array}{l}\text { Reduced dose } \\
(n=118)\end{array}$ & $P$ value \\
\hline \multicolumn{5}{|l|}{ Timing } \\
\hline Arrival on scene to induction (min) & $24(9)$ & $23(8)$ & $26(9)$ & 0.005 \\
\hline \multicolumn{5}{|l|}{ Pre-RSI fluids and vasopressors } \\
\hline $\mathrm{NaCl} 0.9 \%$ from $\mathrm{EMS}$ crews & 34 (10.6\%) & $10(4.9 \%)$ & $24(20.3 \%)$ & $<0.001$ \\
\hline PRBC and/or plasma & $23(6.2 \%)$ & $0(0.0 \%)$ & $23(19.4 \%)$ & $<0.001$ \\
\hline $\mathrm{NaCl} 5 \%$ & $11(3.4 \%)$ & $4(2.0 \%)$ & $7(5.9 \%)$ & 0.059 \\
\hline Vasopressors & $9(2.8 \%)$ & $2(1.0 \%)$ & $7(5.9 \%)$ & 0.009 \\
\hline \multicolumn{5}{|l|}{ Pre-RSI analgesia and sedation } \\
\hline Fentanyl & $12(3.7 \%)$ & $8(3.9 \%)$ & $4(3.4 \%)$ & 0.805 \\
\hline Midazolam & $64(20.0 \%)$ & $44(21.6 \%)$ & $20(17.0 \%)$ & 0.352 \\
\hline Ketamine & $75(23.3 \%)$ & $48(23.5 \%)$ & $27(23.9 \%)$ & 0.951 \\
\hline Morphine & $14(4.4 \%)$ & $8(3.9 \%)$ & $6(5.1 \%)$ & 0.622 \\
\hline \multicolumn{5}{|l|}{ Preparation } \\
\hline Preoxygenation & $321(99.7 \%)$ & $204(100 \%)$ & $117(99.2 \%)$ & 0.366 \\
\hline Apnoeic oxygenation & $30(9.3 \%)$ & $21(10.3 \%)$ & $9(7.6 \%)$ & 0.428 \\
\hline \multicolumn{5}{|l|}{ Route of administration drugs } \\
\hline IV & $315(97.8 \%)$ & $202(99.0 \%)$ & $113(95.8 \%)$ & 0.053 \\
\hline 10 & $7(2.2 \%)$ & $2(1.0 \%)$ & $5(4.2 \%)$ & \\
\hline \multicolumn{5}{|l|}{ Grade view } \\
\hline 1 & $213(66.2 \%)$ & $133(65.2 \%)$ & $80(67.8 \%)$ & 0.707 \\
\hline II & $86(26.7 \%)$ & $56(27.5 \%)$ & $30(25.4 \%)$ & \\
\hline III/IV & $16(5.0 \%)$ & $12(5.9 \%)$ & $3(2.5 \%)$ & \\
\hline Unknown & $7(2.1 \%)$ & $3(1.4 \%)$ & $5(4.2 \%)$ & \\
\hline \multicolumn{5}{|l|}{ RSI success rate } \\
\hline Desaturation below $90 \%$ at any single moment $(n)$ & $118(36.7 \%)$ & $56(27.5 \%)$ & $62(52.5 \%)$ & $<0.001$ \\
\hline First pass success $(\%)$ & $301(93.5) \%$ & $189(93.1) \%$ & $111(94.1) \%$ & 0.095 \\
\hline Overall success rate $(\%)$ & $321(99.7) \%$ & $189(100) \%$ & $117(99.2) \%$ & 0.366 \\
\hline Surgical airway & 1 & 0 & 1 & \\
\hline
\end{tabular}

Categorical data are reported as frequency (n) and percentage (\%) and numerical data as median (IQR) or mean (SD). Vasopressors (epinephrine, $\mathrm{n}=8$, metaraminol, $\mathrm{n}=1$ ).

IO, intraosseous; ISS, Injury Severity Score; IV, intravenous; PRBC, packed red blood cells; RSI, rapid sequence induction.

\section{DISCUSSION}

In the present study, we demonstrate that a standardised prehospital RSI protocol comprising of fentanyl, ketamine and rocuronium is safe and effective in a heterogeneous population of major trauma patients. However, a (delayed) hypotensive response occurs in a minority of patients, irrespective of the dose regime of induction drugs administered.

Although many prehospital services have already adopted fentanyl, ketamine and rocuronium as their standard regime for prehospital trauma RSI, until now limited data existed on the efficacy and safety of this approach. ${ }^{1011}$ The current study shows that the absolute blood pressure reduction is proportional to the regime chosen: patients receiving a full-dose induction regimen had a larger drop in blood pressure than patients receiving a reduced-dose induction regimen. However, the incidence of hypotensive episodes was around 9\% in both groups. This is equal or lower than reported in various large in-hospital studies, ${ }^{13-15}$ and comparable to hypotension rates reported for prehospital RSI in a system where no fixeddose regimes are used. ${ }^{16}$

The equal incidence of hypotensive episodes in the full-dose and reduced-dose induction regime groups is likely indicative of the fact that physicians on scene often choose a reduced-dose regimen for frail patients or patients in suspected hypovolaemic shock, with less compromising effect on their blood pressure. However, as the physiological reserve of these patients is also less, the incidence of hypotensive episodes equals the incidence 
Table 3 Haemodynamic response to RSI in patients anaesthetised with standardised dosing regimen of fentanyl, ketamine and rocuronium

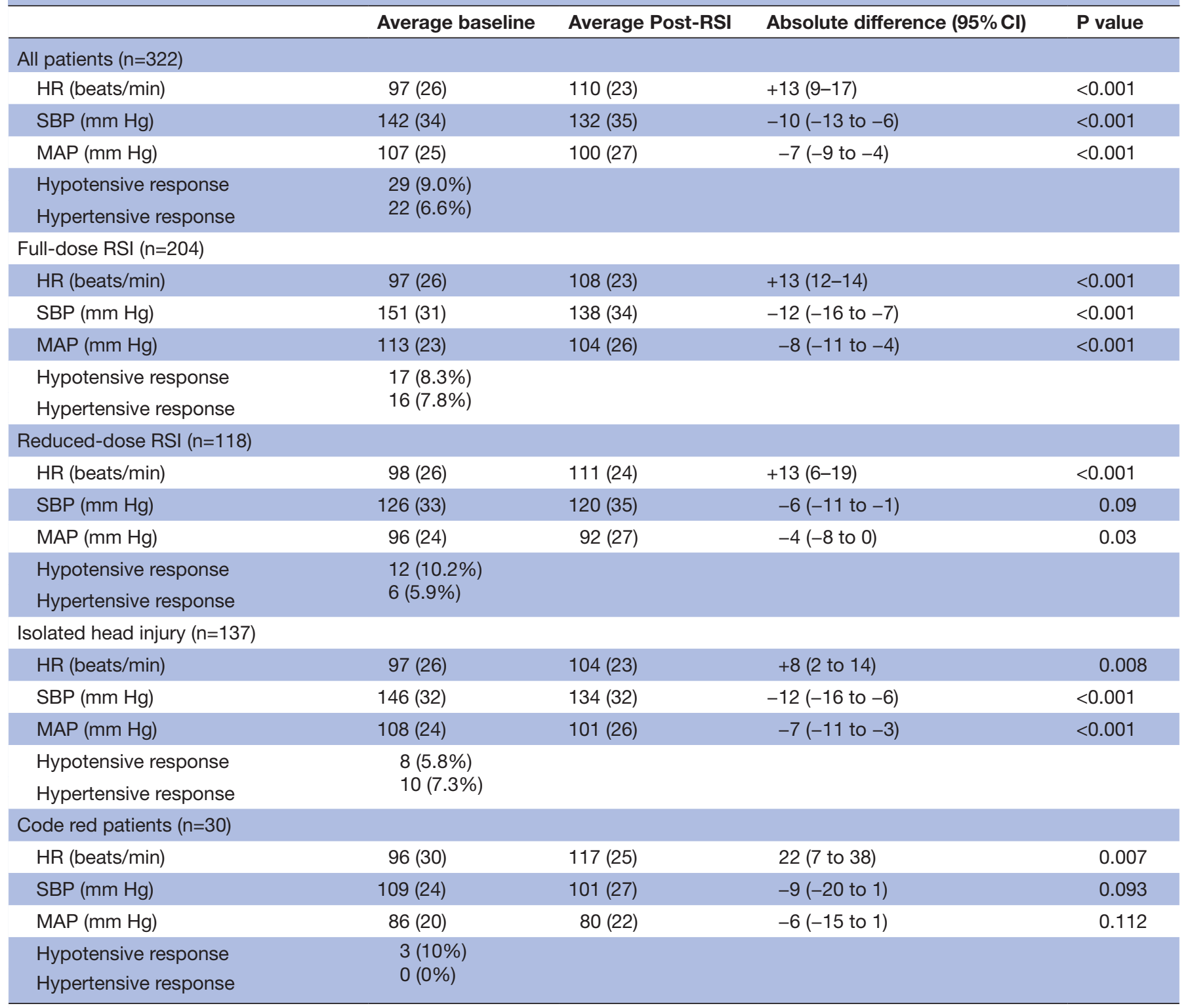

Categorical data are reported as frequency (n) and percentage (\%) and numerical data as mean (SD), or absolute difference (95\%).

MAP, mean arterial pressure; RSI, rapid sequence induction; SBP, systolic blood pressure.

in the full-dose group. Interestingly, around $21 \%$ of the patients receiving a full-dose induction regime had a shock index $>0.8$ prior to induction. Five patients in this group demonstrated a hypotensive episode, and one can speculate if choosing a reduced-dose regime could have avoided this. Sensitivity analysis demonstrated that when physicians on scene made a dose modification from the standard induction dose regimes to account for pre-RSI medication administered, this was not associated with a reduced rate of hypotensive episodes, which supports the fact that safe trauma RSI can be delivered with a limited number of RSI dosing regimes.

Although the incidence of hypotensive episodes was low $(9.0 \%)$, the mean drop in SBP in these patients was as large as $53 \mathrm{~mm} \mathrm{Hg}$ and lasted several minutes. A drop of this magnitude potentially affects cerebral perfusion and may be of clinical consequence, especially in patients with (isolated) head injury. These hypotensive episodes tended to occur predominantly $6-8$ min after the RSI. This was also the moment of lowest average recorded blood pressures. This delayed hypotensive response is compatible with the pharmacological profile of fentanyl: although fentanyl has a relatively short distribution halflife, peak tissue concentrations of intravenous fentanyl are generally not reached until $5 \mathrm{~min}$ after administration. ${ }^{17}$ Anticipation of hypotensive episodes (eg, by readily available vasopressors) and careful observation of haemodynamics is therefore warranted not only immediately after induction, but also later in the post-intubation phase. 


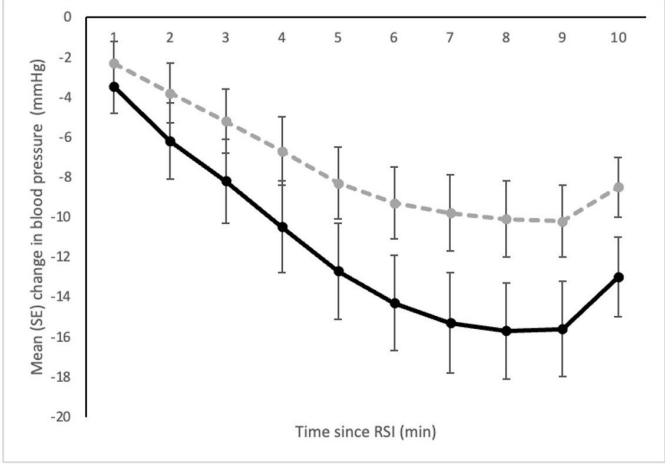

A

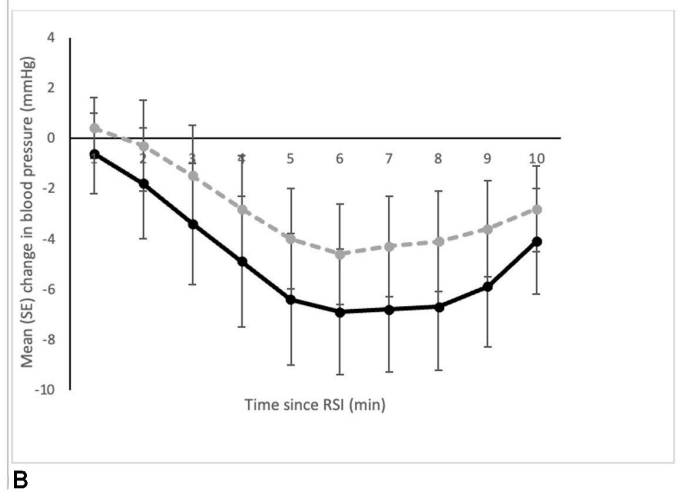

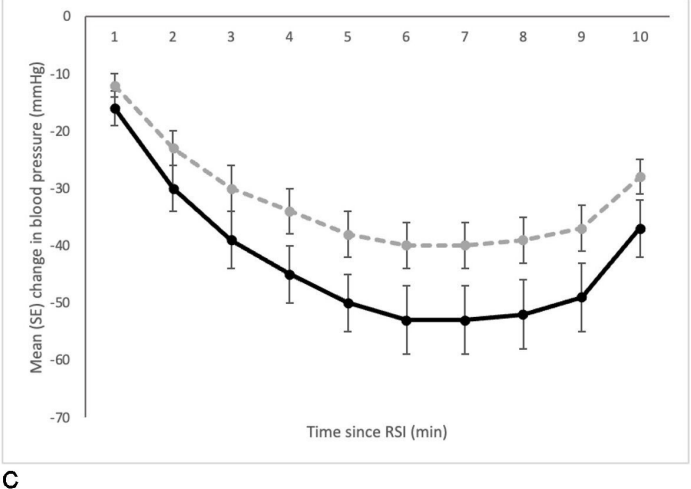

C

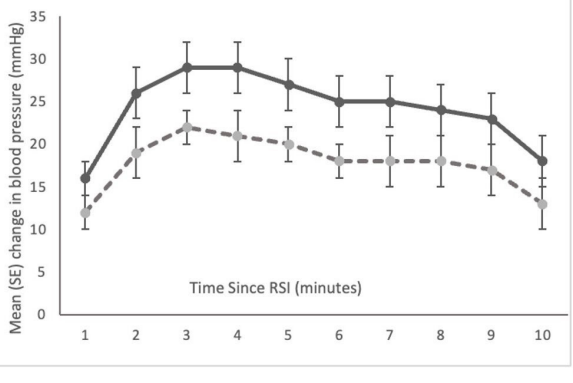

Figure 2 The average haemodynamic response in the first $10 \mathrm{~min}$ post rapid sequence induction (RSI) plotted against time. Black, systolic blood pressure (SBP); grey dashed, mean arterial pressure (MAP). (A) Patients receiving a full-dose regimen $(n=204)$. (B) Patients receiving a reduced dose regimen $R S I(n=118)$. (C) Patients demonstrating a hypotensive response $(n=29)$. (D) Patients demonstrating a hypertensive response $(n=22)$.

Hypertensive episodes pose a significant risk, especially to those with a traumatic brain injury and those with haemorrhage and unstable blood clots. ${ }^{71} 19$ A hypertensive response to laryngoscopy should therefore be avoided in such patients. As fentanyl blunts the sympathomimetic response to laryngoscopy and endotracheal tube placement, it is thought to contribute to the prevention of hypertensive episodes. However, despite the relatively high dose of fentanyl in the full-dose regimen $(3 \mu \mathrm{g} / \mathrm{kg})$, hypertensive episodes post-RSI did still occur, predominantly in the first few minutes after RSI, although the incidence was lower than reported in previous studies. ${ }^{6} 1120$ This may well be the result of the timing of the fentanyl administration. Fentanyl was not administered as premedication, but at the time of the RSI. As laryngoscopy is generally started 45-60 s after RSI drugs are administered, peak tissue concentration of fentanyl at that time may have been insufficient to blunt the sympathetic response to laryngoscopy.

Based on these findings, it might be worth to reconsider the dose and timing of fentanyl administration during prehospital trauma RSI. The results of the FAKT trial, ${ }^{21}$ a randomised double-blind placebo-controlled trial evaluating the impact of fentanyl as an adjunct to ketamine and rocuronium on postintubation haemodynamics, may hopefully provide more guidance on the optimal dose of fentanyl for prehospital RSI in the near future.
Our study has several limitations. First, this is an observational study, not powered to detect any predefined endpoints. Although associations are reported between the medication administered during the RSI process and changes in blood pressures, no causal relationships could be established based on our findings, and our findings are mainly hypothesis generating. Second, our endpoints for hypotensive and hypertensive responses were based on at least two consecutive BP readings to rule out spurious measurements. However, this carries the risk of missing true transient responses of short duration and may thereby result in an underestimation of the true incidences of hypotensive and hypertensive responses. Third, although our study population was quite heterogenous regarding mechanism of injury and injuries sustained, several subgroups where underrepresented. Penetrating injuries were rare, as were patients with significant bleeding, warranting a Code Red response. This is understandable, however, as prehospital anaesthesia has been shown to be related to an adverse outcome in these patients by prolonging time to definitive care. ${ }^{22}{ }^{23}$ Our study only included trauma-RSI's and, as such, results cannot be extrapolated to other populations. Finally, due to the retrospective study design, we had to rely on data completeness. Pre-RSI blood pressures were unavailable in 53 patients, who therefore had to be excluded from analysis, which may have resulted 
in selection bias. Finally, long-term outcome data where incomplete or unavailable for a significant proportion of the study population.

\section{Conclusion}

Although prehospital induction of anaesthesia for trauma with fentanyl, ketamine and rocuronium is not related to a significant change in haemodynamics in most patients, a (delayed) hypotensive response with a clinically significant drop in SBP should be anticipated in a minority of patients irrespective of the dose regimen chosen.

\section{Author affiliations}

${ }^{1}$ Air Ambulance Kent Surrey and Sussex, Redhill, UK

${ }^{2}$ Emergency Medicine, University Medical Centre Groningen, Groningen, The Netherlands

${ }^{3}$ University of Surrey, Guildford, UK

${ }^{4}$ School of Health Sciences, University of Surrey, Guildford, UK

\section{Twitter Ewoud ter Avest @ewoudterAvest}

Contributors EtA and RL conceived the study. DR, JG, MD and SAM collected and verified the data. EtA and DR performed data analysis, and EtA drafted the manuscript. All authors read and approved the final manuscript. EtA accepts full responsibility for the overall content of the paper.

Funding The authors have not declared a specific grant for this research from any funding agency in the public, commercial or not-for-profit sectors.

Competing interests None declared.

Patient consent for publication Not applicable.

Ethics approval This project met National Institute for Healthcare Research (NIHR, UK) criteria for service evaluation and formal ethical approval was therefore not required. The project was approved by the AAKSS Research \& Development Committee.

Provenance and peer review Not commissioned; externally peer reviewed.

Data availability statement Data are available upon reasonable request. Deidentified participant data are available upon reasonable request.

Supplemental material This content has been supplied by the author(s). It has not been vetted by BMJ Publishing Group Limited (BMJ) and may not have been peer-reviewed. Any opinions or recommendations discussed are solely those of the author(s) and are not endorsed by BMJ. BMJ disclaims all liability and responsibility arising from any reliance placed on the content. Where the content includes any translated material, BMJ does not warrant the accuracy and reliability of the translations (including but not limited to local regulations, clinical guidelines, terminology, drug names and drug dosages), and is not responsible for any error and/or omissions arising from translation and adaptation or otherwise.

Open access This is an open access article distributed in accordance with the Creative Commons Attribution Non Commercial (CC BY-NC 4.0) license, which permits others to distribute, remix, adapt, build upon this work non-commercially, and license their derivative works on different terms, provided the original work is properly cited, appropriate credit is given, any changes made indicated, and the use is non-commercial. See: http://creativecommons.org/licenses/by-nc/4.0/.

ORCID iD

Ewoud ter Avest http://orcid.org/0000-0002-1462-6130

\section{REFERENCES}

1 Woodall N, Frerk C, Cook TM. Can we make airway management (even) safer?-lessons from national audit. Anaesthesia 2011;66:27-33.

2 Cook TM, MacDougall-Davis SR. Complications and failure of airway management. Br J Anaesth 2012;109:i68-85.

3 Bowles TM, Freshwater-Turner DA, Janssen DJ, et al. Out-of-theatre tracheal intubation: prospective multicentre study of clinical practice and adverse events. Br J Anaesth 2011;107:687-92.

4 Russotto V, Myatra SN, Laffey JG, et al. Intubation practices and adverse peri-intubation events in critically ill patients from 29 countries. JAMA 2021;325:1164-72.

5 Burgess MR, Crewdson K, Lockey DJ, et al. Prehospital emergency anaesthesia: an updated survey of UK practice with emphasis on the role of standardisation and checklists. Emerg Med J 2018;35:532-7.

6 Lockey DJ, Crewdson K, Lossius HM. Pre-hospital anaesthesia: the same but different. Br J Anaesth 2014;113:211-9.

7 Sherren PB, Tricklebank S, Glover G. Development of a standard operating procedure and checklist for rapid sequence induction in the critically ill. Scand J Trauma Resusc Emerg Med 2014;22:41.

8 Crewdson K, Lockey D, Voelckel W, et al. Best practice advice on pre-hospital emergency anaesthesia \& advanced airway management. Scand J Trauma Resusc Emerg Med 2019;27:6

9 Gawande A. The checklist manifesto: how to get things right. New York: Metropolitan Books, 2010.

10 Lyon RM, Perkins ZB, Chatterjee D, et al. Significant modification of traditional rapid sequence induction improves safety and effectiveness of pre-hospital trauma anaesthesia. Crit Care 2015;19:134.

11 Howes RJ, Walsh J, Le Clerc S, et al. Hypotension following prehospital rapid sequence induction of anaesthesia: a retrospective database analysis. J R Nav Med Serv 2017;103:111-3.

12 von Elm E, Altman DG, Egger M, et al. The strengthening the reporting of observational studies in epidemiology (STROBE) statement: guidelines for reporting observational studies. Lancet 2007;370:1453-7.

13 Reich DL, Hossain S, Krol M, et al. Predictors of hypotension after induction of general anesthesia. Anesth Analg 2005;101:622-8.

14 Südfeld S, Brechnitz S, Wagner JY, et al. Post-induction hypotension and early intraoperative hypotension associated with general anaesthesia. Br J Anaesth 2017;119:57-64.

15 Hefner AC, Swords D, Kline JA. The frequency and significance of post-intubation hypotension during emergency airway management. J Crit Care 2012;27:e9-13.

16 Rognås L, Hansen TM, Kirkegaard H, et al. Anaesthesiologistprovided prehospital airway management in patients with traumatic brain injury: an observational study. Eur J Emerg Med 2014;21:418-23.

17 Peng PW, Sandler AN. A review of the use of fentanyl analgesia in the management of acute pain in adults. Anesthesiology 1999;90:576-99.

18 Perkins ZB, Gunning M, Crilly J, et al. The haemodynamic response to pre-hospital RSI in injured patients. Injury 2013;44:618-23.

19 Morris J, Cook TM. Rapid sequence induction: a national survey of practice. Anaesthesia 2001;56:1090-115.

20 Perkins ZB, Wittenberg MD, Nevin D, et al. The relationship between head injury severity and hemodynamic response to tracheal intubation. J Trauma Acute Care Surg 2013;74:1074-80.

21 Ferguson I, Milligan J, Buttfield A, et al. Fentanyl or placebo with ketamine for emergency department rapid sequence intubation: the FAKT study protocol. Acta Anaesthesiol Scand 2019;63:693-9.

22 Ruelas OS, Tschautscher CF, Lohse CM, et al. Analysis of prehospital scene times and interventions on mortality outcomes in a national cohort of penetrating and blunt trauma patients. Prehosp Emerg Care 2018;22:691-7.

23 Crewdson K, Rehn M, Brohi K, et al. Prehospital emergency anaesthesia in awake hypotensive trauma patients: beneficial or detrimental? Acta Anaesthesiol Scand 2018;62:504-14. 